\title{
Design and Development of Smart Robot Car for Border Security
}

\author{
Khushwant Jain ${ }^{1}$ \\ Centre for Development of Advanced Computing \\ Mohali, Punjab, India
}

\author{
Vemu Suluchana ${ }^{2}$ \\ Centre for Development of Advanced Computing \\ Mohali, Punjab, India
}

\begin{abstract}
The proposed work presents designing and development of a multipurpose smart robot car using wireless camera detecting alive humans, fire, harmful gases, metals, obstacles at remote areas and send information to main location. The proposed system uses machine intelligence to provide immediate response from sensors. The robot system is equipped with sensors those can alert the user when some anomaly appears within the range while robot is working. The main feature of this robot differentiating it from others is execution of versatile tasks in night and rough areas. This whole robot system works in two modes. Mode one is automatic mode and the other is user controllable mode. By default, robot works in automatic mode in which all sensors like human detecting sensor, temperature sensor, gas detecting sensor, metal detecting sensor, obstacle detecting sensor, Ultrasonic sensor are functional for automatic action. The movement of robot in automatic mode is controlled through obstacle detecting sensor and ultrasonic sensor. In user controllable mode, user sends the signal to robot car using RF module and controls it manually. User could watch the surroundings through wireless camera built in the robot car and gives directions to change the path accordingly. In both modes, user could hear the talk of humans at the border areas with the help of microphone in wireless camera.
\end{abstract}

\section{General Terms}

Surveillance, Inspection, Robot Car, Dimensions, Wireless Sensor Network, Program Implementation, Wireless Camera, DC Gear Motor, Acrylic Sheet, automatic mode, User Controllable Mode.

\section{Keywords}

Surveillance, Inspection, Human Detector, Gas Detector, Fire Detector, Metal Detector, Obstacle Detector, Ultrasonic Detector, Wireless Camera, RF Module, Border Areas, Robot Car, Remote Control.

\section{INTRODUCTION}

Webster defines a robot as "An autonomous device that performs functions normally ascribed to humans or a machine in the form human." Generally, it is machine that functions in spite of a living person. Robots are used for special applications like handling hazardous situations and tasks that need high accuracy and speed. A danger event is normally happened by the negligence of humans. To implement real time inspection and surveillance of the border security, intelligent remote monitoring system is developed. Wireless sensor network is used to monitor physical or environment conditions such as temperature, gases, humans, metals etc. Our system consists of sensory network, embedded system and intelligent program on the robot vehicle. Whenever there is any deviation from the original state of sensors occur at robot side (i.e. VEHICLE SIDE) it transmits the signals to remote side (i.e. USER SIDE) where detected quantity showed on LCD. It can also climb terrain at certain angles. This project surely creates revolution in defense systems as a surveillance and inspection robot.

\section{PROPOSED SYSTEM}

Sensory network in our proposed system detect parameters like human presence, harmful gases, pistols, bombs, mines, fire etc at remote areas. In border areas, we know the entry of any human is totally prohibited. Through PIR (Pyroelectric Infrared Sensor) sensor we can detect presence of any human being in prohibited areas. In this proposed system, Metal detection sensor, gas detection sensor, temperature sensor, PIR sensors are used. Metal detector is used for detecting metals at the border areas because mines, bombs, pistols, electronic circuits are made up of metals. Temperature sensor measures the temperature of outside environment [10] and when temperature goes beyond the limits then according to feeded algorithm robot transmit the signals to user side where user is available to take action. Switching from automatic mode to user mode is very fast and from without any delay.

\section{HARDWARE REQUIREMENTS}

\subsection{Microcontroller}

Microcontroller is a general purpose device having large number of the components of a microprocessor system on a single chip. Now a days, a variety of microcontrollers are available in a market we can use any of them. Here, we are using microcontroller ATMEL AT89S51 for this vehicle. It had 128 bytes of RAM, 4K bytes of on-chip ROM, compatible with MCS-51 products, 32 programmable I/O Lines, Programmable Serial Channel, Six interrupt sources, low-power idle and power-down modes.

\subsection{Metal Detector}

The main module of metal detector is the inductive oscillator circuit which detects for high frequency current losses in a coil. Hence, metallic body can be detected with the variations in the high frequency Eddy current losses. Output signal level is altered when a metal object is approached [9].

When supply voltage is given to the sensor then no current flows hence the output of this sensor is $+5 \mathrm{~V}$. If the metal object is close to the searching coil, the output current flows more and output voltage given by sensor is $0 \mathrm{~V}$. On the other hand, the current will decrease when the object is far from the searching coil.

\subsection{Human Detector}

PIR (Pyroelectric sensor) is used for detection of humans. It is a pyroelectric device that detects motion by measuring 
changes in the infrared (heat) levels emitted by surrounding objects. This module consists of Fresnel lens and motion detection circuit. PIR sensor consists of material made up of crystalline that generates electric charge when exposed to infrared radiation. Voltage is generated when striking of infrared radiation on Fresnel lens changes. Fresnel lens focuses the infrared radiations onto the element. The PIR Sensor has a range of approximately 20 feet (6 meters). Output of this sensor becomes high when sudden change in motion occurs [5], [9]. This is used for detection of humans or intruders at border areas.

\subsection{Obstacle Detector}

The principle of this sensor is that Infrared light is send through IR LEDs which is reflected by any object in front of the sensor. In this sensor, instead of constant beam, a pulse of IR light at $1 \mathrm{KHz}$ frequency is transmitted and receiver will detect IR pulses of exact same frequency, rejecting all higher and lower frequencies. Operation range of this sensor varies according to the color of the object; light color object has more range. Range of this sensor is $10 \mathrm{cms}$ for white object. This sensor gives active low output when obstacle is detected [9].

\subsection{Gas Detector}

This sensor is used for detection of harmful gases like LPG, iso-butane, propane, LNG combustible gases at the border areas. This sensor is necessary because when these gases are ignited by sparks it may result into explosion. The output of this sensor is in analog form [9]. According to concentration of gas at outside environment in PPM (parts per million) output of the sensor varies from 0 to $5 \mathrm{~V}$. In normal conditions, output of this sensor is $12 \mathrm{mV}$. The output of this sensor is fed into analog to Digital converter unit [ADC 0804] which is further processed in to digital form before passing it to microcontroller unit. Concentration of gas in air is calculated according to the following equation

$\mathrm{PPM}$ of Gas $=$ Analog Output $(\mathrm{mV}) * 2$

\subsection{Ultrasonic Sensor}

This sensor is used for distance measurements from the obstacles. The output of this is in the form of serial data. So this data is given to the receiver pin of microcontroller which receives data and firstly and then according to algorithm present in microcontroller our robot will move in automatic mode [9].

\subsection{Temperature Sensor}

Temperature sensor (LM35) is used for detection of fire. Output of this sensor is in analog form and varies directly with temperature and it is calibrated directly in Celsius [9]. It is used for fire detection in an area where robot is moving. It detects the temperature of the environment. Range of this sensor is $-55^{\circ} \mathrm{C}$ to $150^{\circ} \mathrm{C}$.

\subsection{Wireless Camera}

Wireless camera will send audio and video signals to user side. The Camera is wired to a wireless transmitter and the signal travels between the camera and the receiver. The wireless camera picture is sent by the transmitter and the receiver collects this signal and outputs it to our Computer or laptop. Wireless camera sends real time audio and video inputs which can be seen on a remote monitor in the base station from where the robot is being controlled by user in user mode. This camera having range of 150 foot (line of sight), providing full motion, real time, color video with no delay. It can easily conceal in box made by us and the built-in microphone provides great sound quality. We used T.V Tuner card for watching the A/V signals on our laptop.

\subsection{RF Module}

This module consists of $433.92 \mathrm{MHz}$ transmitter and receiver modules. Transmitter transmits the signal to receiver module where receiver receives and gives signal to microcontroller. The RF module has been used in conjunction with a set of four channel encoder/decoder ICs. Here HT12E and HT12D have been used as encoder and decoder respectively. The conversion of parallel inputs into serial set of signals is done through encoder. These signals are serially transferred to the receiver side through RF. The decoder is used after the RF receiver to decode the serial format and regain the original signals as outputs. Encoder IC (HT12E) receives parallel data in the form of address bits and control bits. Control signals coming out from sensor outputs along with 8 address bits compose a set of 12 signals in parallel. The encoder HT12E encodes these parallel signals into serial bits for transmission. Here four channel means we give 16 commands to our vehicle side and vehicle also sends 16 signals through wireless communication through RF module. In this we are using four signals to control the four directions of robot car that are forward, left, right and backward direction. These transmitter and receiver are connected to encoder and decoder ICs which encodes or decodes the 12 bits of data ( 8 address and 4 data bits) into serial data which is used to control the directions. Address bits of transmitter and receiver are same that is why when data bit at the transmitter changes then at the receiver that only that particular bit is changed.

\section{PLACEMENT AND DIMENSIONS}

Acrylic sheet is used for the manufacturing of the top of the chassis base for robot vehicle because of unique properties like light weight, more impact resistance, more flexible, excellent resistance to chemicals, insulator, weather resistance and many more advantages.

Tapping is provided at the front side of robot vehicle to avoid damage of sensors which are placed inside the tapping box which is made up of acrylic sheet. Whole robot is painted with dark black color so that intruders can't see the robot. Wireless camera is mounted at below inside the slot in robot vehicle so that user can clearly see the objects in front of it. Batteries and $\mathrm{PCB}$ are placed inside into the box.

Placement and dimensions on sensors are shown in figures: figure1, figure 2 and figure 3 . Figure 1 shows the front view and dimensions of slots for ultrasonic sensor, human detector and wireless camera and metal detector is mounted at the below with the help of clamps.

In figure 2 we shows the top view of robot vehicle in which small slot is provided for temperature sensor and for antennas

Side view of the robot car is presented in figure3. Obstacle sensor, gas detector is placed in the slots given in the figure 3 . 


\subsection{Front view}

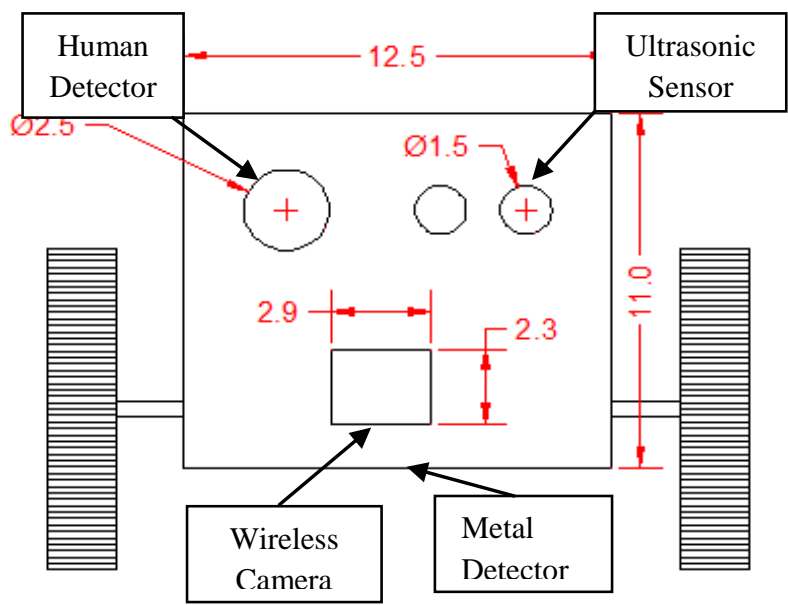

Figure 1: Sensors on the front side

\subsection{Top View}

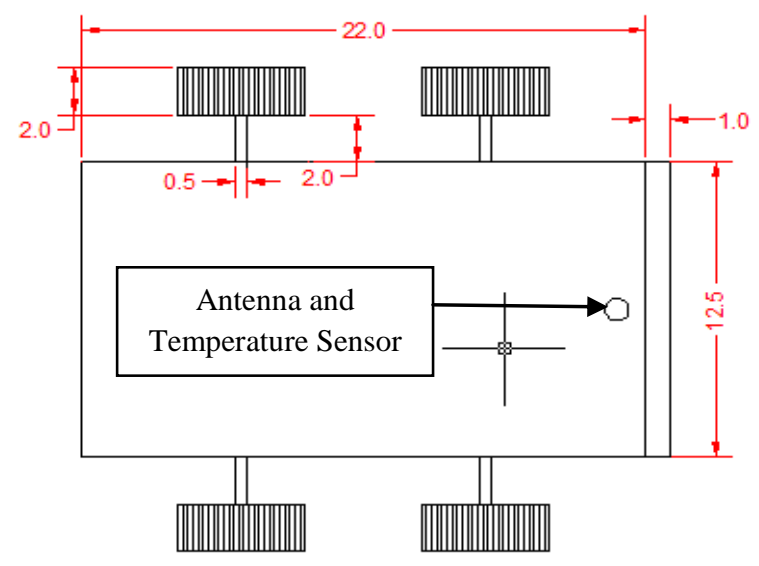

Figure 2: Temperature Sensor and Antenna on Top Side

\subsection{Side View}

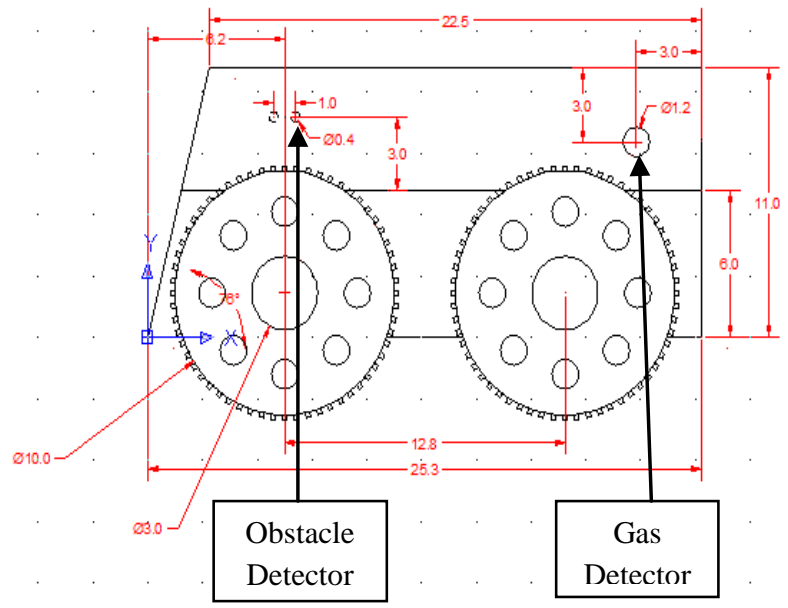

Figure 3: Obstacle Sensor and Gas Sensor on right side

\section{Block Diagram of User Side}

In user side, command keys, LCD, RF module and buzzer are interfaced with Microcontroller. By default, the robot vehicle works in automatic mode [1]. Whenever we want to control it manually we send a signal to vehicle side through the remote keys or command keys interfaced with RF transmitter (1). We give directions to robot vehicle from remote location via watching the output of wireless camera on laptop mounted on vehicle side. Whenever robot gets stuck in unleveled areas and certain rough zones, the user customize the operational ability of the robot vehicle from automatic to manual mode. User also checks for the receiving signals from various sensors those are mounted on vehicle side. For example: when sensors detect metals at remote areas it sends wireless signal via RF Transmitter(2) to user side where 'METAL DETECTED' is shown on LCD and buzzer gives a continuous sound for certain time period. When we want to place the robot in automatic mode we send different command to vehicle side and robot is in automatic mode.

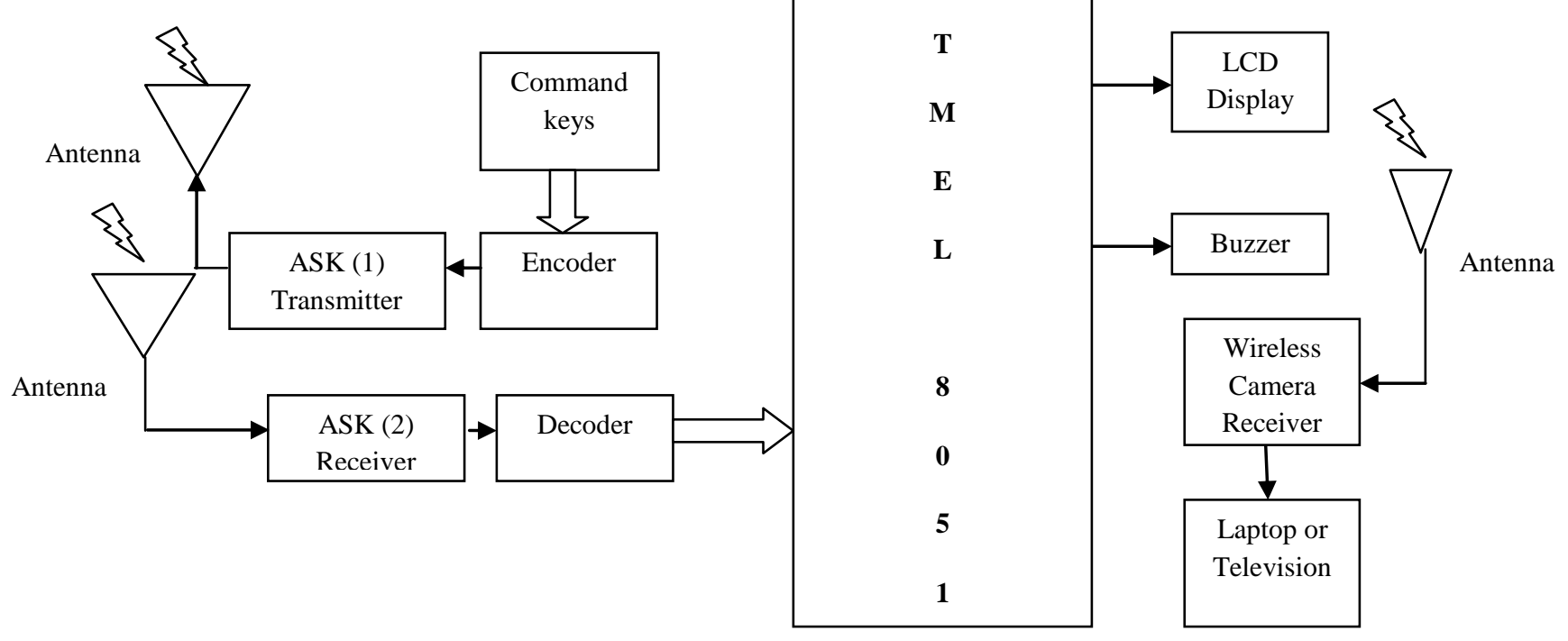

Figure 4: Block Diagram of User Controllable Mode 


\section{BLOCK DIAGRAM OF VEHICLE SIDE}

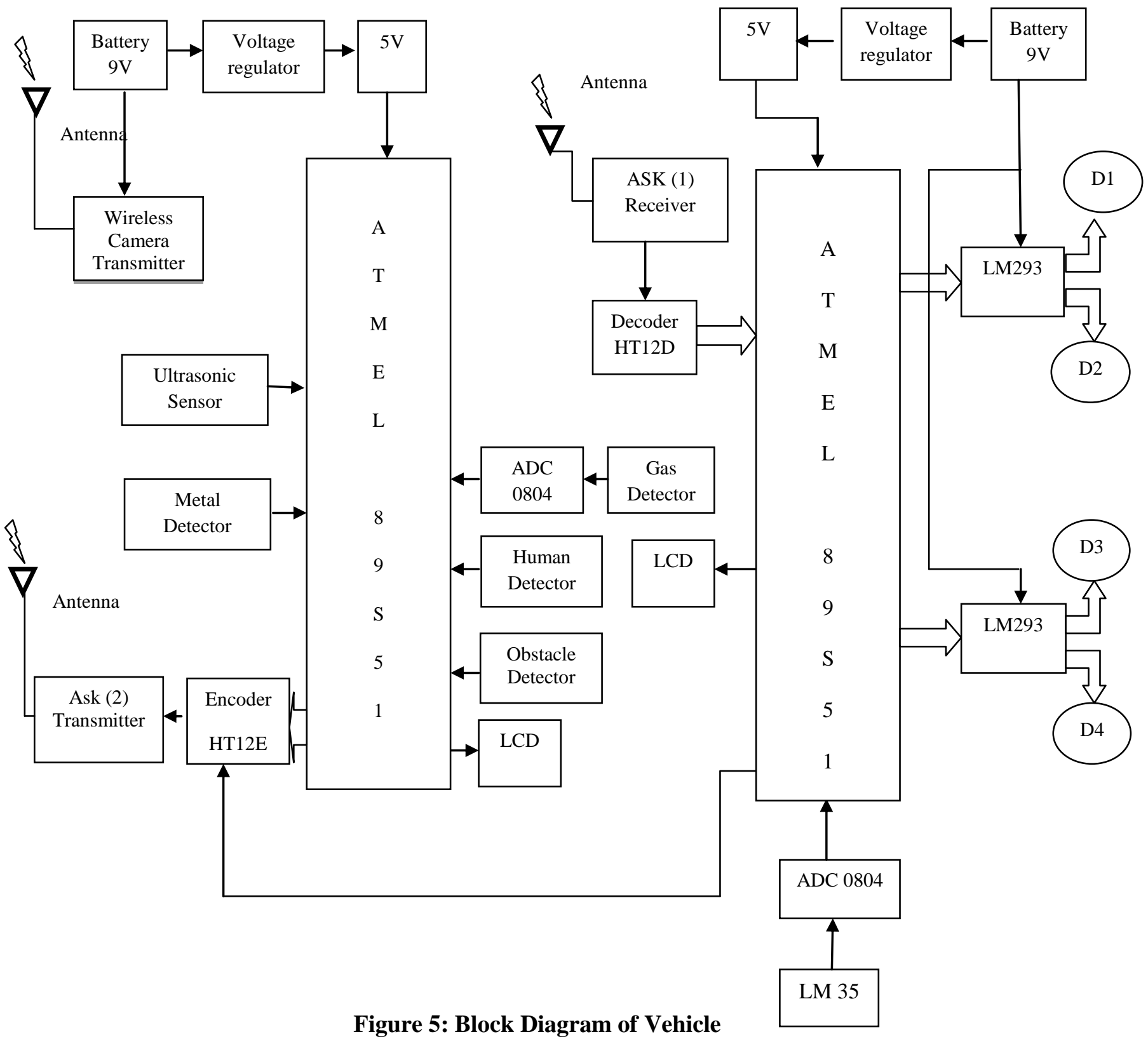

In vehicle side all the Sensors, RF transmitter (2) and receiver (1), LCD and DC motors are interfaced with Microcontroller. Two microcontrollers are used in this side and algorithms for both are different. Four signals are used by us for receiving parallel output from four sensors independently. Once the metal, gas, fire or human is detected, this side transmits the signal wirelessly towards the user side via RF transmitter. Out of the four receiving signals at user side, microcontroller displays the sensing quantity in the LCD and the user will takes action accordingly. For example, when it detects human beings at the inspection area then user can send the troop of soldiers to the place where robot is present to check them. This is working in two modes [1]:

\section{Automatic Mode \\ 2. User Controllable Mode}

In automatic mode, obstacle sensor and the ultrasonic sensor are the two main sensors which are used for movement of the robot. Ultrasonic sensor is attached in the front side of robot as shown in figure 1 and Obstacle sensor is attached at the right side of robot shown in figure 3. Both the sensors works in parallel whenever there is any obstacle present in their paths they send the signals to microcontroller and depends upon algorithm in microcontroller robot will move. When there is any obstacle present in the fixed range of ultrasonic sensor in forward direction and not present in right side of robot, it will move towards right side. Range of both the sensors is different. When there is an obstacle present in right side of robot and in the forward side then it will move towards 
the left side. User also watches the robot through wireless camera from the remote location. Most important thing is that other sensors works normally in both modes. When sensors find any deviation from normal values they transmit the signal wirelessly to the user side where the detecting physical quantity is displayed on LCD and buzzer will $\mathrm{ON}$ which alerts the user present on that side. Wireless camera sends us real time audio and video signals of areas where robot vehicle is doing its searching operation. We can feed the audio and video in our computer for future purposes.

In user mode, when the user wants to control the robot manually it transmits the signal through remote through RF transmitter to the vehicle side. All sensors metal detecting sensor, human detection sensor, fire detection sensor, gas detection sensor works normally and wireless camera sends the audio and video signals at user side without any delay. Through wireless camera user controls the robot in real time. Sometimes in automatic mode robot get stucked and not able to come out from the searching area the user can control it manually through remote.

\section{FEATURES}

1) PIR detecting sensor detects motions from up to $600 \mathrm{cms}$.

2) Metal detecting sensor detects metals up to $6 \mathrm{cms}$.

3) The detectable range of obstacle sensor is up to $10 \mathrm{cms}$.

4) Ultrasonic sensor detects the obstacle from $400 \mathrm{cms}$.

5) Robot can control from 200 feets through remote with the help of $434 \mathrm{MHz} \mathrm{RF}$ transmitter and receiver module.

6) Robot can work in rough areas and it can climb at certain angles.

7) Wireless camera sends real time audio and video signals at remote location and its range is 150 feets.

8) Ground clearance of our robot is $3.2 \mathrm{cms}$.

\section{IMPEMENTATION}

Algorithms made for user side and vehicle side are different. They are written in embedded $\mathrm{C}$ language. The algorithm for the robot vehicle side for automatic mode and user side is written in such a way that it can take its own decisions and user can control it manually when needed and can place it in an automatic mode for default operations.

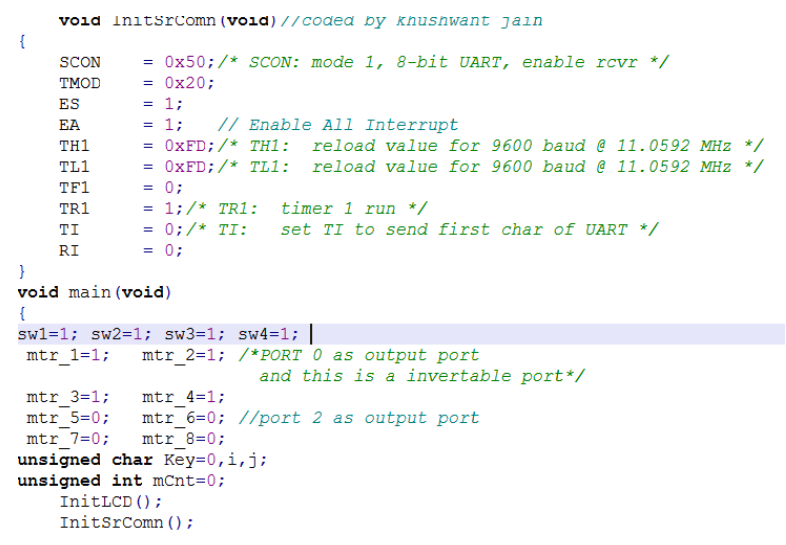

\section{PROS AND CONS}

- Our robot can work in rough areas.

- It is best for surveillance and inspection purposes.

- Our robot can send real time audio and video signals at user side without any delay.

- Our robot is fully autonomous and also controlled through remote but its range is 500 feets.

- Our robot can detects humans, metals, harmful gases, fire at border areas.

- It is a low cost and low speed robot which is best of border security. We can use multiple of these robots at border security.

- Disadvantage of this robot is that range of controlling the robot is short and fixed.

\section{RESULTS AND SNAPSHOTS}

In this, we are showing the results and some of the snapshots of project at Vehicle Side and User Side. Figure 6 and figure 7 shows the vehicle side of robot which is ready to sense any physical or non physical quantity. Figure 6 shows robot in stationary position and figure 7 shows robot in working position in a ground at night. Figure 10 shows the outputs at user side when robot detects parameters like human presence, metals (pistols, bombs, mines etc.,), harmful gases and fire at remote locations.

Figure 8 and figure 9 shows the user side of robot where user is operating the vehicle through remote and output of the sensors also display in this side.

In figure 9 and figure 10, we showed the independent module of metal detecting sensor at vehicle side and when it detect metal it sends signal to user side where metal detected is showed on LCD and user took action according to sensing quantity.

This robot vehicle has many advantages as compared to other robots. For instance, take an inspector bots toughbot surveillance and inspection robot available on robot shop [6]. Cost of our robot is INR 15000 as of inspector bot has $\$ 4990$. Our robot vehicle consists of multiple sensors, wireless camera and also works in two automatic and manual mode.

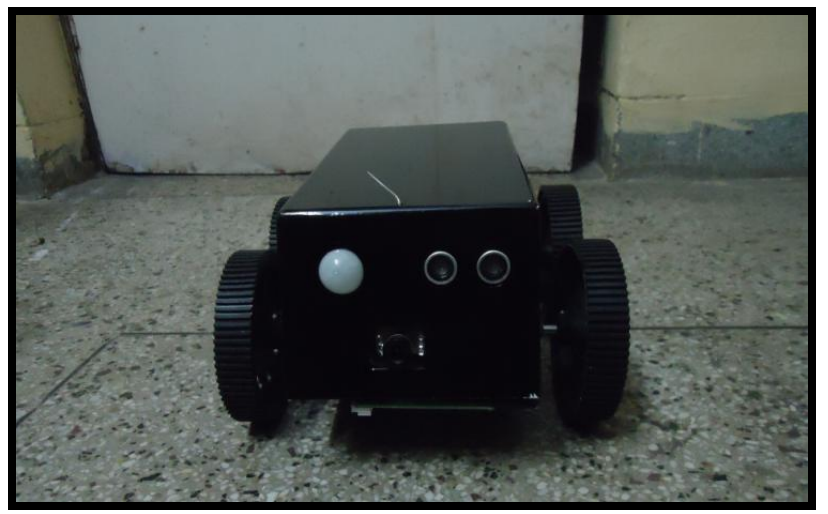

Figure 6: Robot in Stationary position 


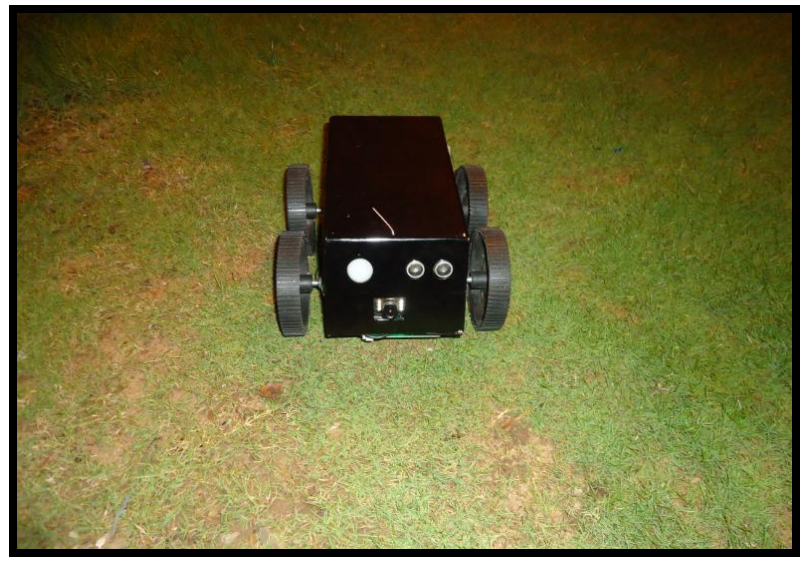

Figure 7: Robot in automatic mode at night

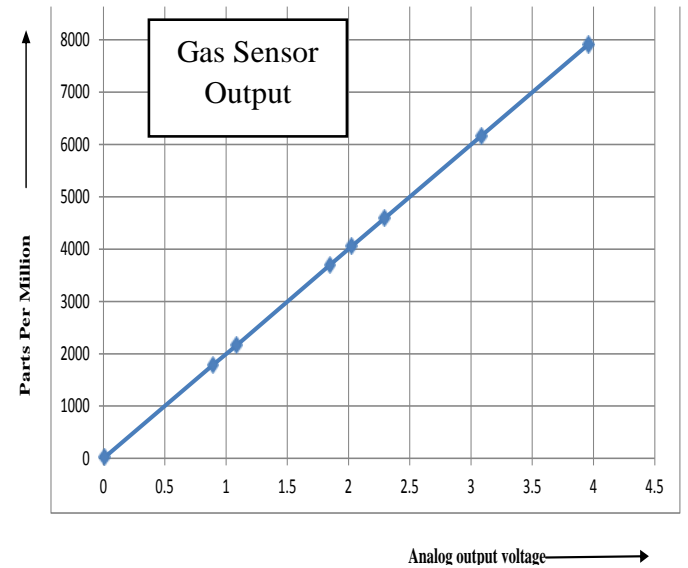

Graph 1: Showing Results taken with respect to After Shave Lotion

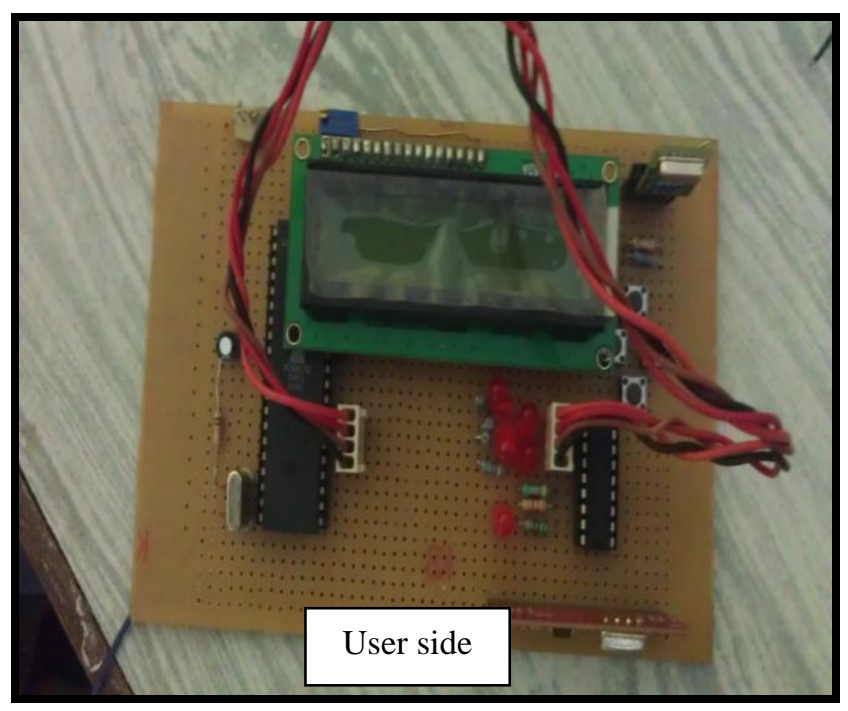

Figure 8: User side

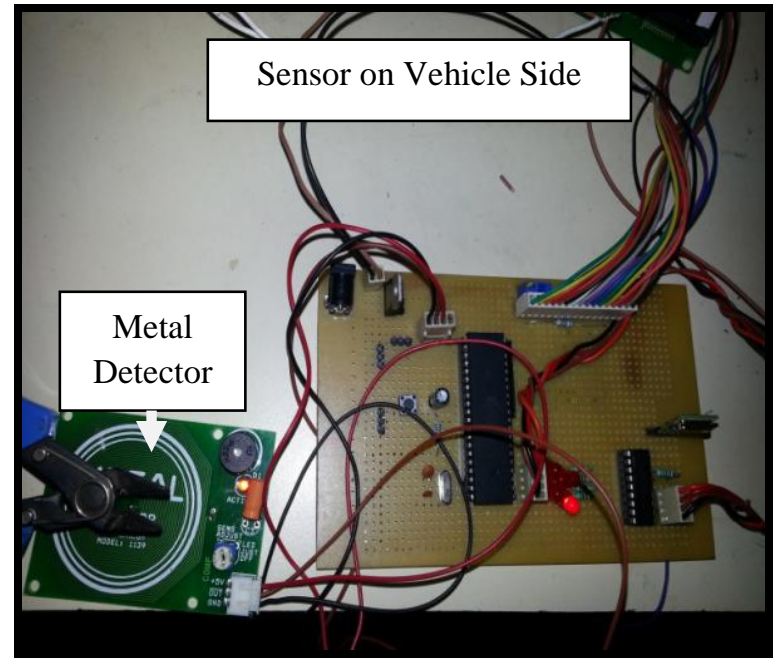

Figure 9: Metal detecting sensor detects Metal

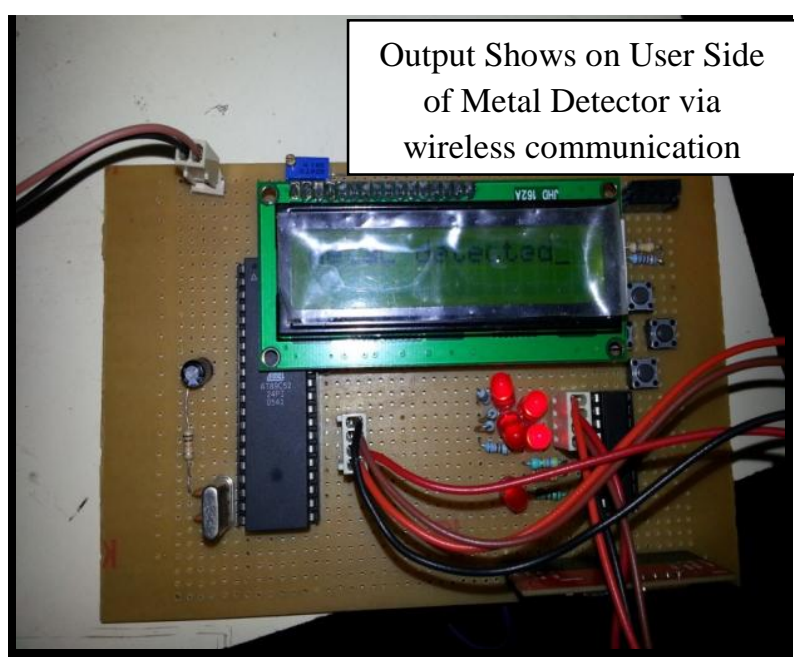

Figure 10: Output shows at User Side
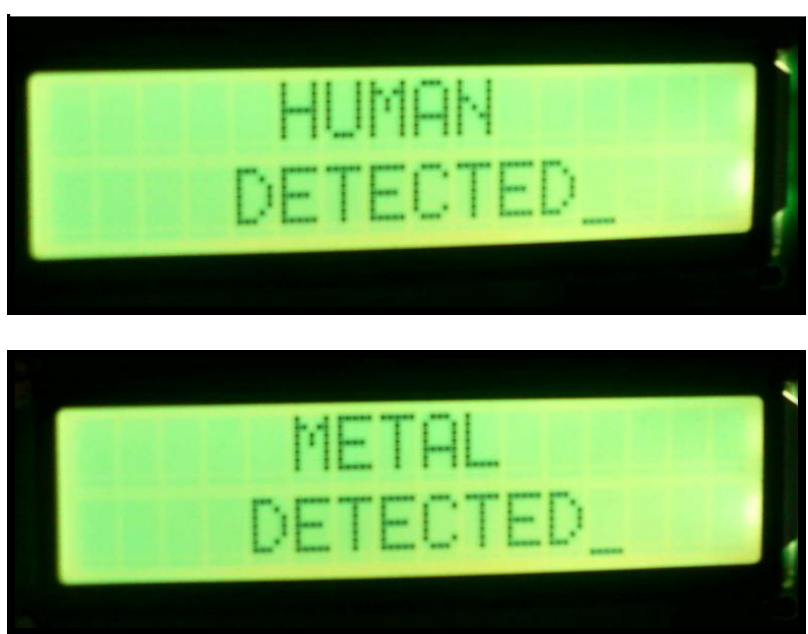

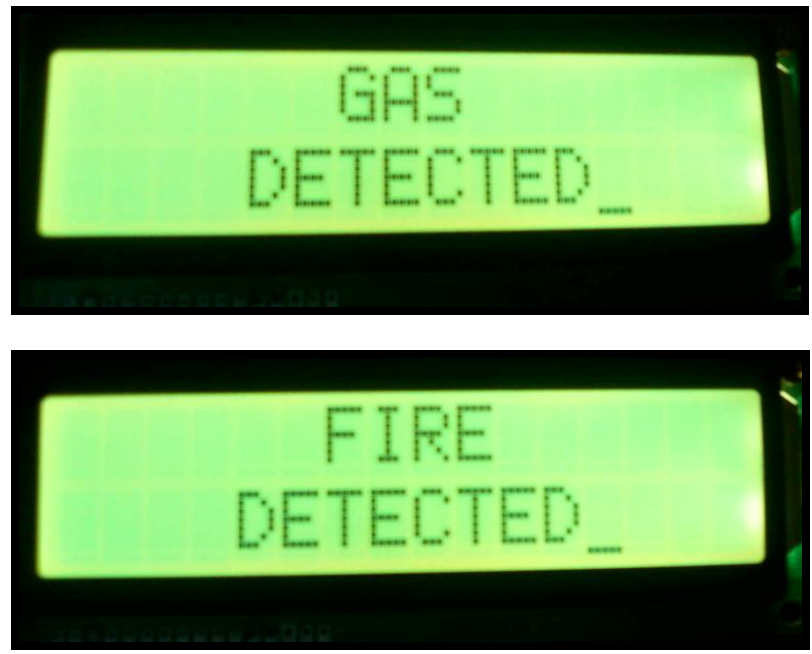

Figure 10: Sensor shows sensing parameters on User Side

\section{ACKNOWLEDGEMENTS}

We would like to extend a special thanks to C-DAC, Mohali, India for providing us means to carry out our research work and provide funds for this project work. We are also grateful to MHRD, Govt. of India for providing us a platform to do our research work.

\section{REFERENCES}

[1] L. Srinivasavaradhan, G. Chandramouli and A.G. Maniprashanna, 2009. $7^{\mathrm{TH}}$ sense. A multipurpose robot for military. MEMSTECH 5th International Conference on Perspective Technologies and Methods in MEMS Design.

[2] C. Marques, J.Cristovao, P.Lima, I.Ribeiro, R.Ventura, J.Frazao. 2006. RAPOSA: Semi-Autonomous Robot for Rescue Operations, Intelligent Robots and Systems,IEEE/RSJ International Conference.
[3] Hou Tsan Lee, Wei Chuan Lin, Ching Hsiang Huang, Yu Jhih Huang. 2011 "Wireless Indoor Surveillance Robot," SICE Annual Conference Waseda University, Tokyo, Japan.

[4] S.Naskar, S. Das, A.K Seth, A. Nath. 2011. Application of Radio Frequency Controlled Intelligent Military Robot in Defense. Communication Systems and Network Technologies (CSNT), International Conference.

[5] Luo, R.C., Chou, Y.T., Liao, C.T., Lai, C.C., Tsai, A.C. 2007. NCCU Security Warrior: An Intelligent Security Robot System. Industrial Electronics Society, 2007. IECON 2007. 33rd Annual Conference of the IEEE.

[6] http://www.robotshop.com/inspectorbots-toughbotsurveillance-inspection-robot.html.

[7] YuanYuan Li; Parker, L.E. 2008. "Intruder detection using a wireless sensor network with an intelligent mobile robot response," Southeastcon, IEEE.

[8] http://www.sunrom.com/

[9] http://www.acrylicsindia.com/products/acrylicsheets.htm

\section{AUTHOR's PROFILE}

${ }^{1}$ Khushwant Jain is pursuing Masters of Technology at CDAC Mohali in Embedded Systems. He completed his Bachelor of Engineering degree in Electronics \& Communication Engineering from Punjab Technical University, Jalandhar in 2010. His research interests include Embedded Systems Design and Robotics. His email id is khushwant9999@gmail.com

${ }^{2}$ Vemu Sulochana has obtained her Bachelor of Technology degree from JNTU Kakinada and Master of Technology degree from NIT, Hamirpur in 2004 and 2009 respectively. In 2011, she joined C-DAC, Mohali to conduct innovative research in the area of VLSI design, where she is now a Project Engineer - II. Her email-id is vemus@cdac.in 